\title{
Konversationsanalyse und diskursive Psychologie
}

\section{Entstehungsgeschichte und disziplinäre Einordnung}

Dieser Text behandelt die Konversationsanalyse (KA) als eine breit etablierte Methodologie zur Untersuchung von sozialen Interaktionen und die diskursive Psychologie (DP), welche sich spezifisch damit befasst, wie psychologische Phänomene in der sozialen Interaktion zum Ausdruck gebracht und thematisiert werden. Zwar war die KA ursprünglich nur eine von mehreren Quellen für die Entstehung der DP, doch hat sich die DP in den letzten Jahren methodologisch zunehmend der KA angeglichen, sodass sie heutzutage als eine Art „KA psychologischer Phänomene“ betrachtet werden kann und deshalb hier gemeinsam mit der KA dargestellt wird.

Die KA wurde von Harvey Sacks in den frühen 1960er Jahren begründet (Sacks 1992). Sacks war an der University of California, Berkeley und Irvine, tätig. Er war ein Schüler des Mikrosoziologen Erving Goffman, mit dem ihn das Interesse an der Untersuchung sozialer Interaktionen als nach eigenen Regeln organisierten Ereignissen verband. Intellektuell knüpfte Sacks jedoch mehr an die Ethnomethodologie Harold Garfinkels (1967; Heritage 1984) an, die sich für die Methoden interessiert, die Gesellschaftsmitglieder benutzen, um handelnd soziale Ordnung herzustellen und soziale Handlungen zu verstehen. Die Ethnomethodologie nahm ihrerseits wesentliche Impulse von der Sozialphänomenologie Alfred Schütz' (1981[1932]) auf, der die Konstitution der sozialen Welt darin begründet sah, wie Akteure ihrem Erleben und Handeln Sinn verleihen. Während Schütz wie der Begründer der Phänomenologie, Edmund Husserl, diese Leistungen in allgemeinen Strukturen der Tätigkeit des Bewusstseins des Subjekts suchte, wandte sich die Ethnomethodologie beobachtbaren Handlungen von Akteuren (z.B. beim Dokumentieren und Kategorisieren von Ereignissen) zu, und sie versuchte, grundlegende Erwartungen und Regeln des sozialen Handels durch die Untersuchung der Reaktion auf deren Durchbrechung in sog. breaching experiments (Krisenexperimenten) zu identifizieren (Garfinkel 1967). Die KA radikalisiert diese Empirisierung des Forschungsgegenstands, indem sie das Programm einer ,naturalistischen Soziologie“ propagiert, d.h., einer ausschließlich auf Audio- und Videodaten gestützten Untersuchung des Verhaltens von Gesellschaftsmitgliedern in natürlichen Situationen (Sacks 1984).

Wie der Name „Konversationsanalyse“ anzeigt, stand zunächst die Untersuchung der ordinary conversation, d.h., alltäglicher, nicht-zweckgerichteter Interaktionen (v.a. Telefongespräche) zwischen Bekannten und Freunden, im Vordergrund. Schon Sacks' frühe Untersuchungen befassten sich aber auch mit institutionellen Daten (z.B. telefonische Suizidberatung, Gruppentherapie). Dieser Forschungsstrang ist in den letzten Jahrzehnten immer bedeutsamer geworden (vgl. Drew \& Heritage 1992; Heritage 2005a). Folglich ist es angemessener, den generellen Gegenstand der KA als talk-in-interaction zu bezeichnen (s. 
Schegloff 1991). Damit ist auch schon gesagt, dass die KA sich im Unterschied etwa zu Goffmans primärem Interesse an der nonverbalen Interaktion (1974) und zu den ethnomethodologisch inspirierten workplace studies (Knoblauch \& Heath 1999) für verbal dominierte Interaktionen interessiert. In den letzten Jahren ist allerdings die videogestützte Untersuchung der multimodalen Interaktion, die neben der verbalen Interaktion auch Blick, Gestik, Mimik, Körperpositur und -bewegungen, die Benutzung von Objekten im Kontext der Interaktion und andere kommunikativ relevante visuelle Verhaltensformen einbezieht, immer wichtiger geworden (s. Heath et al. 2010; Schmitt 2007). Seit Mitte der 1990er Jahre hat sich aus der KA als weitere gegenwärtig besonders prosperierende Forschungsrichtung die sog. „Interaktionale Linguistik“ (Selting \& Couper-Kuhlen 2000) entwickelt. Sie befasst sich damit, wie in der Interaktion sprachliche Formen verwendet und mit ihnen interaktive Strukturen hergestellt werden. In diesem Forschungskontext ist auch die Prosodie zu einem zentralen, über das Verbale hinausgehenden Forschungsgegenstand geworden (Couper-Kuhlen \& Ford 2004).

In Deutschland wurde die KA durch die Schriften von Kallmeyer und Schütze (1976) sowie Jörg Bergmann (1981) bekannt. Kallmeyer und Schütze befassten sich mit unterschiedlichen Ordnungsebenen der Interaktionskonstitution, so z.B. mit den Verfahren der Sachverhaltsdarstellung im Kontext von Beschreibungen und Erzählungen (Kallmeyer \& Schütze 1977) und mit der Handlungsorganisation von Beratungsgesprächen (Kallmeyer 1985). Bergmanns Studien bezogen sich auf klassische Gegenstände der KA wie die Organisation des Turntakings im Kontext von Pausen und Schweigen (1982), erweiterten sich aber bald zur Perspektive auf kommunikative Gattungen wie Klatsch (1987). Das Konzept der „kommunikativen Gattungen“ (s. Günthner \& Knoblauch 1994), das Bergmann zusammen mit dem Wissenssoziologen und Schütz-Schüler Thomas Luckmann entwickelte und das neben der KA auch vor allem auf die Ethnografie der Kommunikation (SavilleTroike 1989) zurückgriff, ist vielleicht der wichtigste genuin deutsche Beitrag zur KA.

In Deutschland wird, etwa im Unterschied zu Skandinavien und Japan, nur sehr selten „rein“ konversationsanalytisch geforscht. Die unterschiedlichen Spielarten der deutschen "Gesprächsanalyse“" kombinieren die KA vor allem mit der Kontextualisierungstheorie (Gumperz 1982) und mit Goffmans Konzepten der rituellen Ordnung der Interaktion (Goffman 1971) oder der Interaktionsbeteiligung (Goffman 1981), greifen in unterschiedlicher Weise aber auch auf andere Konzepte aus Pragmatik, Ethnografie und Soziolinguistik zurück (s. Auer 1999). Während die KA ihren Ursprung in der Soziologie hatte, ist sie in Deutschland wie in vielen anderen Ländern mittlerweile mehr in der Linguistik beheimatet (vgl. Deppermann 2007).

Die discursive psychology (DP) entstand seit Mitte der 1980er Jahre in England, ausgehend von der wissenssoziologischen Diskursanalyse (Potter \& Wetherell 1987). Ihre Grundidee besteht darin, psychologische Phänomene nicht mehr als innerpsychische, kognitive oder emotionale Phänomene zu verstehen, sondern als Phänomene des Diskurses zu untersuchen. Ein entscheidender Ausgangspunkt war dabei die konstruktivistische Auffassung des Philosophen Wittgenstein, derzufolge sich die Bedeutung und die intersubjektive Realität psychischer Phänomene nach der Verwendung der Ausdrücke, mit denen diese bezeichnet werden, richten. Neben sprachphilosophischen Quellen integrierte die DP zunächst v.a. Ansätze aus der Diskursanalyse und der Rhetorik, aber auch aus der Ethnomethodologie und der KA (s. Edwards \& Potter 1992). Im Unterschied zur KA arbeitet die DP nicht nur mit Daten verbaler Interaktionen, sondern auch mit schriftlichen Quellen. 
Während die KA vor allem an der formalen Organisation des Handelns in Interaktionen interessiert ist, beschäftigt sich die DP besonders mit der Konstruktion von Darstellungen und Begründungen von Handlungen. Dabei geht die DP weniger detailgenau als die KA vor, die Beachtung interaktiver Reaktionen und Konsequenzen (vgl. Abschnitt 3) spielt methodologisch eine weit geringere Rolle als in der KA, während gedankenexperimentellen Verfahren und der Berufung auf soziale Wissensbestände eine größere Rolle zukommt. In den letzten 15 Jahren hat sich die DP allerdings zunehmend der KA angenähert (vgl. Wooffitt 2005), sodass viele der neueren Veröffentlichungen als eine Spielart der KA verstanden werden können, die sich spezifisch damit beschäftigt, wie psychische Phänomene in der Interaktion thematisiert und welche sozialen Handlungen damit vollzogen werden.

\section{Theoretische und methodologische Prämissen und Grundannahmen}

Unsere Alltagswelt besteht zu einem großen Teil aus verbalen Interaktionen. In ihnen werden die für unser Erleben und unsere Handlungen relevanten Deutungen der sozialen Welt zum Ausdruck gebracht, verhandelt und modifiziert. In Interaktionen wird die soziale Ordnung dessen, was für uns der Fall ist, was wahr und richtig ist, wie wir zueinander stehen, wer wir selbst sind und wie wir miteinander umgehen, hergestellt. Interaktionen zeichnen sich durch folgende Grundeigenschaften aus:

- Sequenzialität: Interaktionen bestehen aus einer Abfolge von aufeinander folgenden Beiträgen (Schegloff 2007). Soziale Ordnung besteht daher in einer prozessualen Vollzugswirklichkeit (Bergmann 1981), soziale Strukturen werden also durch die Systematik der Abfolge von Ereignissen konstituiert und sind entsprechend zu beschreiben.

- Interaktivität: Jeder folgende Beitrag ist sowohl kontextbezogen, d.h., er ist zugeschnitten auf einen vorangehenden Beitrag, dessen Verständnis er zugleich dokumentiert, und er schafft einen neuen Kontext, der die Vorgabe für Anschlusshandeln ist und hinsichtlich dessen Erwartungen (Projektionen) erzeugt (Heritage 1984, S.242). Soziale Wirklichkeit entsteht daher intersubjektiv, in Prozessen wechselseitigen Deutens und Verstehens.

- Pragmatizität: In den Sequenzen von Handlungen, aus denen Interaktionen bestehen, werden sowohl kollektive Gesprächszwecke (z.B. ein Problem klären, sich verabreden, einen Witz erzählen) als auch individuelle Ziele der Gesprächsteilnehmenden (z.B. eine für sich günstige Lösung erzielen, jemanden überzeugen, als ein guter Unterhalter anerkannt werden) bearbeitet (Kallmeyer 2005).

- Konstitutivität: Das Gesprächsverhalten der Interaktionsteilnehmer/innen wird weder von psychologischen noch von soziologischen Variablen und Regeln determiniert, sondern die Teilnehmenden produzieren ihre Gesprächsbeiträge in Bezug auf die jeweiligen Gesprächskontexte, -aufgaben und -zwecke. Dabei verdeutlichen sie, welche psychologischen und sozialen Sachverhalte (z.B. Identitäten, Rollen, soziale Beziehungen, institutionelle Tatsachen) für die Interaktion in welcher Weise relevant und für deren Ablauf folgenreich sind (Schegloff 1991).

- Methodizität: Die Interaktionsteilnehmer/innen benutzen mehr oder weniger konventionelle Interaktionspraktiken, die zum Vollzug spezifischer Aktivitäten eingesetzt werden (Schegloff 1997). Interaktionspraktiken bestehen aus (z.B. sprachlichen) Formen, 
die für bestimmte Funktionen bzw. zur Bearbeitung bestimmter Aufgaben im Gespräch in bestimmten Kontexten eingesetzt werden können. Ihre Anwendung kann mehr (z.B. bei Begrüßungen) oder weniger (z.B. bei rhetorischen Praktiken) durch kontextgebundene Erwartungen geregelt sein.

- Materialität: Da Interaktionen (zumeist) mündlich stattfinden, sind sie flüchtig, und wenn sie face-to-face stattfinden, sind sie multimodal. Die medialen Bedingungen der Gesprächsbeteiligung (face-to-face, Telefon, Chat, TV usw.) und die je nach medialer Bedingung einsetzbaren materialen, körperlichen Ressourcen bestimmen die Aufgaben und Möglichkeiten des Handelns und der inter- und intrasubjektiven Koordination von Verhalten in der Interaktion (Deppermann \& Schmitt 2007).

Die KA ist ein induktiver, datengetriebener Forschungsansatz. Sie lehnt es ab, a priori Hypothesen aufzustellen. Sie fordert dagegen, Forschungsfragestellungen und zentrale theoretische Begriffe aus der Analyse von Interaktionsdaten selbst zu entwickeln. Dabei greift sie auf den Bestand an vorliegenden Forschungen und empirisch fundierten Begriffsprägungen zur Organisation von Interaktionen zurück. Zentral ist der Anspruch, analytische Begriffe und Befunde in den Daten zu verankern, d.h., in einer rekonstruktiven Analyse zu zeigen, dass und wie die wissenschaftlichen Begriffe als Explikationen der Eigenschaften und Funktionen des lokalen Gesprächshandelns aufgefasst werden können. Im Mittelpunkt steht dabei für die KA die Einnahme einer „Teilnehmerperspektive“. Dies bedeutet (entgegen einem weit verbreiteten Missverständnis) nicht, über Motive und Intentionen der Interaktionsteilnehmenden zu spekulieren, sondern es beinhaltet die Anforderung zu zeigen, dass sich diese in ihrem Handeln an einer bestimmten interaktiven Aufgabe orientieren. Bei der Analyse muss also nachgewiesen werden, dass ein aus Sicht der Forscher/innen relevantes Problem im Gespräch auch von den Teilnehmenden in einer erkennbaren Weise systematisch bearbeitet wird oder dass ein Kontextsachverhalt (wie eine bestimmte soziale Zugehörigkeit) von ihnen im Gespräch erkennbar relevant gemacht und folgenreich zur Geltung gebracht wird (Schegloff 1997). Grundsatz der analytischen Mentalität der KA ist Sacks' Maxime: „order at all points“ (Sacks 1984). Sie beinhaltet, dass jedes auch noch so zufällig, rätselhaft oder unsinnig erscheinende Detail einer Interaktion als sinnhaft motiviert, d.h., als methodisch produziertes Phänomen mit einer (zu entdeckenden) Funktion und Bedeutung für die Gesprächsteilnehmenden zu behandeln und entsprechend systematisch zu rekonstruieren ist (vergleichbar den Prämissen der objektiven Hermeneutik, s. Bergmann 1985). Die KA folgt damit einer problemtheoretischen und funktionalen Sicht von Interaktion, d.h., jedes Interaktionsphänomen wird als Ausdruck einer Orientierung an der Bearbeitung bzw. Lösung von Interaktionsaufgaben und -problemen verstanden (vgl. Kallmeyer 2005). Diese können selbstverständlich neue Probleme und Aufgaben nach sich ziehen, für die wiederum Routinen der Problemlösung bereitstehen können. Die KA hat die Aufgabe, die funktionale Motivation, das entsprechende Potenzial zur Problemlösung und zur Schaffung neuer Probleme und die Art und Weise, wie Interaktionsteilnehmende die entsprechende Praktik einsetzen und verstehen, aufzuweisen. Dabei ist aber stets der Interaktionsprozess, d.h., sowohl der vorangehende wie der folgende Interaktionskontext, das entscheidende methodologische Kriterium.

Die KA ist von ihren methodologischen Prämissen her antimentalistisch, d.h., der Rekurs auf Intentionen und - in geringerem Maße - auf Wissen der Teilnehmenden gilt nicht als adäquate Erklärung für beobachtbares Interaktionshandeln, da Mentalitätszuschreibun- 
gen ontologisch, als psychologische Sachverhalte nicht abzusichern sind, und da ihr reines Gegebensein sie noch nicht zu für den konkreten Interaktionsprozess wirksamen Faktoren macht. Außerdem geht es der KA nicht um die Rekonstruktion von psychologischen Motiven und Determinanten für Interaktionen, sondern um die Analyse der Regeln, Praktiken und Erwartungsstrukturen, die in Bezug auf Interaktionen als eigenständige Ebene sozialer Wirklichkeit selbst gelten. Im Gegensatz etwa zur kritischen Diskursanalyse, zur objektiven Hermeneutik oder zu psychoanalytischen Ansätzen enthält sich die KA jeder Bewertung, ob die von den Gesprächsteilnehmenden vollzogenen Handlungen moralisch gut, sinnvoll, zweckmäßig, gesund oder krank usw. sind (Prinzip der „ethnomethodologischen Indifferenz", s. Garfinkel \& Sacks 1976).

\section{Methodisches Vorgehen}

Die KA arbeitet ausschließlich mit „natürlichen Daten“, d.h. mit Audio- und (zunehmend) Videoaufnahmen von nicht eigens für Forschungszwecke arrangierten sozialen Interaktionen. Ursprünglich ging es der KA dabei gar nicht um die Untersuchung von Gesprächsstrukturen als solchen. Ton- und Videoaufzeichnungen wurden vielmehr zu Methoden der Wahl, da nur mit ihnen die ,passiv registrierende“ Dokumentation des sozialen Handelns in seiner originären Form als flüchtiger Vollzugswirklichkeit möglich ist (Bergmann 1985). Im Unterschied zu allen anderen, in der qualitativen Psychologie und Soziologie gängigen, „rekonstruktiven“ Daten (Bergmann 1985) wie Fragebögen, Interviews oder Feldnotizen bewahren nämlich Audio- und Videoaufnahmen die prozessualen Details des sozialen Geschehens. Im Gegensatz zu anderen Datenerzeugungsmethoden sind sie nicht durch sekundäre Sinnbildungsprozesse der selektiven Erinnerung, der Codierung und der Interpretation der Interviewten bzw. Forschenden überformt, aufgrund derer Merkmale der interessierenden Situation unauflöslich mit den durch die Dokumentation entstehenden Merkmalen konfundiert sind.

Audio- und Videodaten, die aus einem Interaktionsfeld (z.B. einer oder mehreren Arztpraxen, von einer peer group) stammen oder die im Kontext der Bearbeitung einer Fragestellung gesammelt wurden, sind Bestandteile eines „Korpus“. Das Analyseziel besteht in der detailgenauen sequenzanalytischen Untersuchung von alltäglichen und institutionellen Interaktionen. Dazu ist es nötig, die Aufnahmen nach Konventionen zu transkribieren, die sicherstellen, dass die Besonderheiten der gesprochenen Sprache (wie Abbrüche, Korrekturen, nicht-lexikalische Laute, Intonation) und der Verlauf der Interaktion (wie Sprecher/innenwechsel, Überlappungen, Pausen) genau notiert werden. Die ursprüngliche KA-Notation stammt von Sacks' Schülerin Gail Jefferson (in Atkinson \& Heritage 1984). Im deutschen Sprachraum haben sich die Konventionen des Gesprächsanalytischen Transkriptionssystems (GAT) durchgesetzt, die eine verfeinerte und systematischere Prosodienotation erlauben (Selting et al. 2009; vgl. Deppermann \& Schütte 2008). Der folgende Ausschnitt aus einem verhaltenstherapeutischen Gespräch, in dem ein Patient über seinen AIDS-kranken Partner spricht, zeigt ein Beispiel einer Transkription nach GAT:

\#1 Verhaltenstherapie Freiburg Ewald 13:36-14:05; Pat = Patient; Thr = Therapeut

01 A: (.) er hat mittlerweile schon so viel DuRCHgmacht, 02 (.) un: ; 


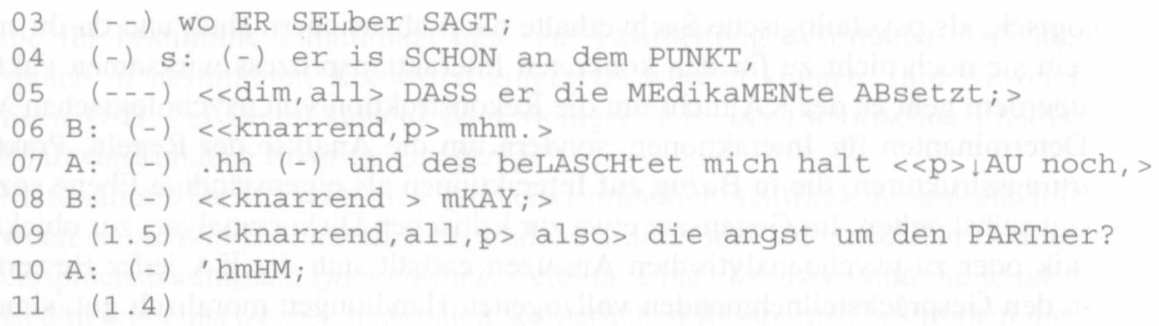

Im Unterschied zu einer alltagsweltlichen Mitschrift ist dieses Transkript in sog. Intonationsphrasen gegliedert. Es enthält die genaue Abfolge der Sprecher und den präzisen Wortlaut, einschließlich aller Abbrüche und Selbstkorrekturen (Zeile 04), nicht-lexikalischer Laute (Zeilen 06 und 10), hörbarem Einatmen (Zeile 07: .hh), Pausen (gemessen, wenn länger als eine Sekunde, mit Punkt, einem, zwei oder drei Geviertstrichen markiert, wenn unter einer Sekunde), Dehnungen (Doppelpunkt), Akzenten (Großschreibung), Intonation am Ende der Intonationseinheiten (Satzzeichen) und Notationen von Tempo (z.B. all für „schnell gesprochen"), Lautstärke (z.B. $p$ für „leise", dim für „leiser werdend") und Stimmqualität (z.B. knarrend). Die KA basiert ihre Erkenntnisse stets auf Analysen der originalen Daten und ihrer Transkription, kodierte bzw. aggregierte Daten werden nicht analysiert (siehe zu Transkription auch den Beitrag von Dresing \& Pehl in diesem Band).

Die Analyse wird durch zwei komplementäre Strategien bewerkstelligt: die detaillierte Sequenzanalyse am Einzelfall und die Arbeit mit Kollektionen (s. Deppermann 1999, Kap. 6). Die detaillierte Sequenzanalyse folgt dem unique adequacy-Kriterium (Psathas 1995), d.h. der Anforderung, dass jedes Detail einer untersuchten Interaktionssequenz in die Untersuchung miteinbezogen und lückenlos gezeigt werden muss, wie die genaue Abfolge der einzelnen Aktivitäten als systematischer, schrittweise aufeinander bezogener Prozess der Sinnbildung und Bearbeitung interaktiver Aufgaben verstanden werden kann. Die Strenge der Analyse liegt also gerade darin, dass Forschende die Daten nicht einfach vorab definierten Kategorien zuordnen und dabei Nichtpassendes passend machen oder ignorieren, sondern zeigen müssen, wie jedes - und zwar auch zunächst unverständlich oder unmotiviert erscheinende - Detail des Handelns (wie z.B. eine Pause, eine unpassend erscheinende Wortwahl, eine Selbstreformulierung) systematisch auf den sich entfaltenden Gesprächsprozess reagiert und zu ihm in bestimmter sinnhafter Weise beiträgt. Entscheidendes Kriterium für die Erstellung und für die Validität der Analyse ist dabei zum einen der Aufweis, wie eine bestimmte Aktivität in Bezug auf einen gegebenen Gesprächskontext lokal, d.h. in Bezug auf den unmittelbar vorangehenden Beitrag, produziert wird, auf welche seiner Aspekte sie bezogen ist, wie sie diese interpretiert, wie sie selbst durch den vorangegangenen Kontext bereits vorbereitet und evtl. gar gefordert worden ist. Zum anderen ist zu zeigen, welche Funktion die Aktivität für den weiteren Gesprächsverlauf hat. Entscheidend sind dabei vor allem die unmittelbar folgenden Reaktionen der Rezipient/innen in den nächsten Gesprächsbeträgen (next turn proof procedure, Sacks, Schegloff \& Jefferson 1974), mit denen diese ihr Verständnis des vorhergegangenen Beitrags zu erkennen geben, sowie die daran anschließenden Reaktionen der Produzent/innen der interessierenden Aktivität in der sog. „dritten Position“ (third position, vgl. Schegloff 1992). Dort nämlich ist zum Ausdruck zu bringen bzw. der Sprecher/die Sprecherin kann stets so verstanden werden, wie er/sie das Verständnis der Ko-Interaktant/innen des eigenen vorangegangenen Beitrags selbst 
versteht und ob er/sie dieses teilt (vgl. Deppermann 2008). Nun wird auch deutlich, worin die "Teilnehmerperspektive" der KA besteht: Sie wendet die zentralen Konstitutionseigenschaften der Interaktion (s. Abschnitt 2: Sequenzialität, Interaktivität etc.) methodologisch, d.h., sie benutzt die grundlegenden Prinzipien des lokal situierten Produzierens und Verstehens von Beiträgen zur Interaktion als Grundlage für die Entwicklung und Legitimation ihrer analytischen Methodik. Diese muss stets dem Kriterium genügen, die Systematik der Interaktion, so wie sie für die Beteiligten beobachtbar produktions- und verstehensrelevant ist, explizit zu machen.

Die detaillierte Sequenzanalyse erstreckt sich auf Einzelfälle aus Kollektionen von Interaktionsausschnitten. Kollektionen werden phänomen- und fragestellungsbezogen gebildet. Typischerweise gibt es zwei alternative Ansatzpunkte:

1. Es ist von Interesse, wie eine bestimmte Aufgabe bzw. ein Problem konversationell bearbeitet wird (= funktionaler Ausgangspunkt, z.B. Erteilung einer Diagnose im ArztPatient-Gespräch; Bearbeitung von Dissens im Streitgespräch), und es wird nach den dafür relevanten Praktiken, ihren jeweiligen Einsatzbedingungen, Folgen, Chancen und Risiken gesucht, d.h. nach dem Variationsspektrum, den Unterschieden und den grundlegenden, gemeinsamen Strukturen des Interaktionsproblems.

2. Im Fokus ist der konversationelle Einsatz einer bestimmten Form bzw. Aktivität (= formaler Ausgangspunkt; z.B. Verwendung einer sprachlichen Form wie jaja oder eine Aktivität wie Blickabwendung von dem Gesprächspartner/der Gesprächspartnerin), und es wird nach ihren Einsatzbedingungen und Funktionen gefragt, eventuell für unterschiedliche Interaktionsaufgaben und in Abhängigkeit von Realisierungen der Form oder Aktivität.

Eine Kollektion beginnt mit der Sammlung aller zunächst potenziell relevant erscheinenden Kandidaten innerhalb eines Datenkorpus (Deppermann 1999, Kap. 6.5). Im Verlauf detaillierter Sequenzanalysen einzelner Fälle werden sukzessive materialgestützte Hypothesen über die wesentlichen Strukturen einer Interaktionspraktik, d.h., über den Zusammenhang ihrer formalen und funktionalen Eigenschaften und ihrer Einsatzbereiche in der Interaktion, aufgestellt, geprüft und verfeinert. Maßgeblich dafür sind komparative Analyseverfahren, wie z.B. der Vergleich mit marginalen Fällen bzw. benachbarten Praktiken, mit abweichenden Realisierungen, die Aufschluss geben über zugrunde liegende Erwartungen und die Reaktion auf deren Verletzung, und die systematische Testung von Annahmen durch die Suche nach Fällen, die bestimmte Merkmale (nicht) aufweisen. Der Prozess der Kollektionsanalyse oszilliert, ähnlich den komparativen Verfahren und dem theoretical sampling der Grounded-Theory-Methodologie (Glaser \& Strauss 1967), beständig zwischen Einzelfall und Kollektion, wodurch die Kollektion mittels Tilgungen, Erweiterungen und Untergliederungen nach und nach bereinigt und systematisiert wird, bis sie sich stabilisiert und eine Struktur der Praktik resultiert, die durch die Detailanalyse weiterer Fälle nicht mehr modifiziert wird. Die resultierende Kollektion repräsentiert also schließlich empirisch die Struktur der Praktik, d.h., ihre Varianten, Einsatzkontexte, Folgen usw. Typisch für diesen Prozess des Arbeitens mit Kollektionen ist daher nicht nur der sukzessive Gewinn von Erkenntnissen über Interaktionspraktiken im Verein mit der sukzessiven Bestimmung der dafür relevanten Daten, sondern oft auch die Veränderung und Eingrenzung der Untersuchungsfrage in einer vorab nicht vorherzusehenden Weise. Die auf die Interaktionsrealität 
passende Fragestellung ist so ,from the data themselves“ (Schegloff \& Sacks 1973, S.290) entwickelt, d.h., die Formulierung der in den Daten zu untersuchenden Probleme und Aufgaben und der dafür angemessenen Begriffe reflektiert selbst schon einen beträchtlichen Teil der Gegenstandserkenntnis, welche nicht durch apriorische theoretische Spekulation zu gewinnen ist.

Im Unterschied zu vielen anderen Ansätzen der qualitativen Sozialforschung steht die KA dem Einbezug von „Kontextwissen“ (über ethnografische, soziale, politische, biografische u.a. Rahmenbedingungen der Interaktion) in der Analyse sehr skeptisch gegenüber. Annahmen über Kontextbedingungen verführen zu interpretativen Kurzschlüssen, d.h., zu vorschnellen vermeintlichen Erklärungen des Interaktionshandelns aus Kontextbedingungen anstelle des detaillierten Aufweises, wie dieses Handeln produziert wird und auf welche Relevanzen sich die Interaktionsteilnehmenden dabei beobachtbar beziehen. Wenn sozialstrukturelle Kontexte wie soziale Identitäten, institutionelle Rollen, hierarchische Beziehungen etc. für die Interaktion wichtig scheinen, dann ist zu zeigen, wie Interaktionsteilnehmende einander verdeutlichen, dass diese Größen für sie relevant sind und dass sie ihr Handeln an ihnen ausrichten (Schegloff 1997). Allerdings gibt es innerhalb der KA unterschiedliche Auffassungen, wie autonom die Organisation von Interaktionen als eigene Ebene der sozialen Organisation gegenüber makrosozialen und kulturellen Ebenen ist (vgl. Schegloff 2005; Levinson 2005), wie manifest und explizit Kontextfaktoren in der Interaktion aufscheinen müssen, um als relevante Orientierungen für Interaktionsteilnehmende gelten zu können (Schegloff 1997; Wetherell 1998), wie viel auf kulturelles Wissen gestützte und nicht „aus den Daten“ bezogene Interpretation unabdingbar in Konversationsanalysen eingeht und in welcher Weise ethnografisches Hintergrundwissen (v.a. bei der Untersuchung von fremden Kulturen und Lebenswelten) unabdingbar für die KA ist (Deppermann 2000).

\section{Grundlegende Strukturen der verbalen Interaktion}

Die grundlegende Einheit von Gesprächen ist der Turn (Gesprächsbeitrag), der seinerseits aus einer oder mehreren aufeinander folgenden turn constructional units (Beitragskonstruktionseinheiten) aufgebaut ist (Sacks et al. 1974; Levinson 1990, S.295ff.). Beitragskonstruktionseinheiten sind gestalthafte Einheiten, die durch das Zusammenspiel prosodischer, syntaktischer, semantischer und pragmatischer Merkmale gebildet werden und mit deren Abschluss signalisiert wird, ob ein Sprecher/innenwechsel erfolgen soll (Selting \& CouperKuhlen 2000). Die Regeln für die Organisation des Sprecher/innenwechsels an transition relevance places, die in alltagsweltlicher, nicht-institutioneller Interaktion gelten, wurden im wohl bekanntesten Artikel der KA beschrieben (Sacks et al. 1974). Sie erklären nicht nur, warum die Sprecher/innenwahl in Gesprächen überwiegend recht reibungslos abläuft, sondern auch, warum und wie es zu Überlappungen und Pausen, zur Produktion von Turnergänzungen oder zu Abbrüchen in bestimmten Interaktionskontexten kommt. Hier wie in vielen anderen KA-Untersuchungen werden somit Phänomene, die zunächst regellos erscheinen und intuitiv nicht zugänglich sind, als systematische Formen der Bearbeitung grundlegender Regel- und Erwartungsstrukturen in der Interaktion verständlich gemacht. Bereits im 2. Abschnitt wurde Sequenzialität als eine Grundeigenschaft von Interaktionen benannt: Das Verhältnis des zeitlichen Benachbartseins (nextness, Schegloff 2007) ist in 
seiner Bedeutung für Interaktionen kaum zu überschätzen, bildet doch ein jeweils gegebener Gesprächsbeitrag den primären Kontext für den nächsten Beitrag und meist auch spezifische Erwartungen, denen dieser Rechnung zu tragen hat und deren Verständnis er qua default dokumentiert. Auf diese Weise ist das Verhältnis der nextness die zentrale Ressource der Interaktionsorganisation, es ist sowohl für die erwartungsbasierte Produktion von folgenden als auch für die lokale Interpretation der vorangehenden Handlungen grundlegend. Diese Erkenntnis unterscheidet die KA fundamental von allen kontextfreien Ansätzen der Handlungsanalyse (wie der Sprechakttheorie, z.B. Searle 1971) und der Inhaltsanalyse: Nicht Handlungen, sondern Handlungssequenzen bilden den grundlegenden Gegenstand der Betrachtung! Besonders offensichtlich ist die Systematik der nextness den sog. „Nachbarschaftspaaren“ (adjacency pairs, Schegloff 2007) eingeschrieben. Dies sind elementare Handlungssequenzen, die aus einer ersten Handlung (z.B. einer Frage) eines Sprechers/einer Sprecherin bestehen, welche eine zweite unmittelbar folgende Handlung eines anderen Sprechers/einer anderen Sprecherin konditionell relevant macht (z.B. eine Antwort). Wie die zweite Handlung ausfällt bzw. ihr Ausbleiben wird in Abhängigkeit von der Art der ersten Handlung interpretiert, so wie umgekehrt die zweite eine implizite Deutung der ersten beinhaltet. Vielfach wird nicht nur ein bestimmter Typ von Folgehandlung erwartbar gemacht (,,projiziert“, Auer 2005), sondern die erste Handlung etabliert eine Präferenz für eine bestimmte Form der Realisierung der zweiten, im Gegensatz zu anderen, dispräferierten Reaktionsmöglichkeiten (Levinson 1990, S.331ff.). So präferiert z.B. eine Einladung als erste Handlung ihre Annahme als Reaktion, eine Ablehnung wäre zwar auch konditionell relevant, aber dispräferiert. Präferenzen sind nicht als individuelle, psychologische Vorlieben zu verstehen, sondern als soziale Erwartungsnormen (Heritage 1995), die dementsprechend zumindest prototypischer Weise damit einhergehen, dass die präferierte Alternative knapp, direkt und ohne weitere Begründung produziert wird, während die dispräferierte meist mit Begründung, oft indirekt und verklausuliert, verzögert, mit Selbstkorrekturen und Abbrüchen durchsetzt produziert wird (Pomerantz 1984).

Die KA hat sich weiterhin ausführlich mit der Organisation von Interaktionen im Ganzen befasst. So wurden sowohl die Eröffnung von Interaktionen (klassischerweise Telefongespräche, s. Schegloff 1968; neuerdings aber auch multimodale Interaktionen, s. Mondada \& Schmitt 2010) als auch deren Beendigung (Schegloff \& Sacks 1973) sowie der Übergang zwischen einzelnen Aktivitätssequenzen und Themen untersucht. Dabei geht es der KA stets darum, die Routineverfahren zu identifizieren, mit denen Gesellschaftsmitglieder rekurrente Interaktionsaufgaben und -probleme lösen, und die sie von der Notwendigkeit entlasten, für jede Interaktion immer wieder neue Lösungen finden zu müssen, die dann entsprechend prekär, da hinsichtlich ihrer Aufnahme und Interpretation durch die Partner/innen ungewiss, wären. Der Fokus der KA richtet sich also im Unterschied zu anderen qualitativen, z.B. psychoanalytischen, marxistischen, objektiv hermeneutischen oder kritisch-diskursanalytischen Verfahren nicht in erster Linie auf Krisen und Konflikte, sondern auf die funktionierende und intersubjektiv zugrunde gelegte Ordnung des Interagierens. Krisen und Zusammenbrüche (z.B. in Form von Missverständnissen) sind dann weniger um ihrer selbst willen oder in kritisch-aufklärerischer, (sozial-) therapeutischer Hinsicht interessant, sondern weil sie über die im funktionierenden Fall stumm und unsichtbar bleibenden Normalitätserwartungen und die Relevanz unscheinbarer Praktiken Aufschluss geben, die erst im Fall ihres Versagens bzw. Fehlens deutlich werden. Dies heißt nun keineswegs, dass mangelnde Aufmerksamkeit, schlechtes Hören, Missverständnisse, Dissens, Koordina- 
tions- und Sprachproduktionsprobleme, strategisches oder provokatorisches Handeln und andere Fälle, in denen die Ordnung der Routine versagt, außerordentlich selten sind. Im Gegenteil, die Robustheit der alltäglichen wie auch institutionellen Interaktion besteht gerade darin, dass für wiederkehrende Probleme auch ebenso routiniert einsetzbare Reparaturverfahren zur Verfügung stehen, die dazu führen, dass Probleme schnell gelöst und als solche meist gar nicht bewusst wahrgenommen werden (Schegloff, Jefferson \& Sacks 1977; Egbert 2009). Die Durchsetzung konversationsanalytischer Transkripte mit Abbrüchen, Wortsuchen, Reparaturen und anderen ,unordentlichen“ Phänomenen, die viele, die sich erstmals mit Transkripten befassen, schockiert und ein Verfremdungserlebnis des Alltäglichen hervorruft, zeigt also weniger, wie chaotisch unsere Interaktionen eigentlich sind, sondern ganz im Gegenteil, wie robust, und dass es trotz aller Widrigkeiten und unvorhersehbarer Einflüsse gelingt, situationsbezogen Sinn herzustellen und Handlungen zu koordinieren.

Bereits eingangs wurde darauf hingewiesen, dass die KA viele Beiträge zur Untersuchung institutioneller Interaktionen erbracht hat. Umfassende Studien liegen vor allem vor zu Expert/innen-Lai/innen-Interaktionen in den Bereichen Arzt-Patient-Gespräche (Heritage \& Maynard 2006; Neises, Ditz \& Spranz-Fogasy 2005), Interaktionen vor Gericht (Atkinson \& Drew 1979), Beratungsgespräche (Nothdurft, Reitemeier \& Schröder 1994), Bewerbungsgespräche (Birkner 2001), Medieninterviews (Heritage \& Clayman 2002), Dolmetschen in verschiedenen institutionellen Situationen (Wadensjö 1992; Martini 2008) oder psychotherapeutische Interaktionen (s. Abschnitt 5). Institutionelle Interaktionen schränken die in alltäglichen Konversationen geltenden Optionen für interaktives Handeln spezifisch ein und haben zugleich spezialisierte Lösungen für die jeweils für sie charakteristischen, in ihnen zu bearbeitenden Interaktionsaufgaben entwickelt (z.B. eine Anamnese erheben, eine Diagnose stellen, eine Verschreibung erklären im Arzt-Patient-Gespräch). Aufgrund ihrer Aufgabenbezogenheit und weil sie oft unter bestimmten rechtlichen, ökonomischen, zeitlichen oder medialen Bedingungen geführt werden, sind sie durch Asymmetrien zwischen den Beteiligten gekennzeichnet. Asymmetrien können in vielen Punkten bestehen: im Fachwissen, im Gebrauch und Verständnis von Fachvokabular und bei den Inferenzmöglichkeiten im Gespräch, in der emotionalen und existenziellen Betroffenheit durch das in der Interaktion behandelte Problem, im Wissen über institutionelle Verfahren und rechtliche Rahmen, bei den Gesprächssteuerungsrechten, in der Verfügung über Sanktionsmöglichkeiten und ökonomische Macht sowie hinsichtlich der Rechte und Pflichten zu bestimmten Arten von Gesprächsbeiträgen. Die KA ist dabei ebenso daran interessiert, die Möglichkeiten und Probleme der Bearbeitung spezieller institutioneller Aufgaben in der Interaktion zu rekonstruieren als auch das ,intuitive“ professionelle Wissen von institutionellen Agenten, wie es sich in ihrem situierten Handeln zeigt; ebenso geht es um Handlungsspielräume und Zwänge der Klient/innen der Institution. Seltener, aber in wachsendem Maße finden sich auch Untersuchungen, die sich in der Tradition der workplace studies mit vollprofessionellen Interaktionen innerhalb von Organisationen oder zwischen Expert/innen befassen.

Die KA hat sich in ihren Untersuchungen vornehmlich formalen und „oberflächennahen" Ebenen der Interaktionsorganisation gewidmet. Da sie es ablehnt, mit mächtigen theoretischen Vorannahmen an die Analyse zu gehen, vermeintlichem Wissen über ethnografische, kulturelle und soziale Tatsachen als Determinanten für Interaktionsverhalten grundsätzlich misstraut und dem Hintergrundwissen der Forschenden einen methodisch eher 
problematischen Stellenwert einräumt, hat sie sich weniger mit den stärker inhaltlich und interpretativ konstituierten Dimensionen von Interaktionen befasst. Die DP setzt dagegen ihren Schwerpunkt in der Erforschung der rhetorischen Verfahren und der interpretativen Strategien, mit denen die Faktizität von Ereignissen konstruiert und die Wahrheit und Glaubwürdigkeit von Darstellungen abgesichert und umkämpft wird (vgl. Edwards \& Potter 1992; Potter, Edwards \& Wetherell 1993; Deppermann 1997; siehe Abschnitt 5.2). Die DP hat gezeigt, dass und wie scheinbar bloß deskriptive Darstellungen von Ereignissen und Handlungen so verfasst werden, dass dabei systematisch Schlussfolgerungen hinsichtlich der Bewertung von Handlungen und Fragen von Motiven, Schuld, Verantwortlichkeit, Interessengeleitetheit und Objektivität bzw. Neutralität der Sprecher/innen und anderer Personen (v.a. Gegner/innen) implizit nahegelegt werden (s.a. Potter 1996; Wooffitt 1992). Die DP befasst sich auch damit, wie Interaktionsteilnehmende Wirklichkeit rhetorisch selektiv konstruieren, und welche Rolle dabei dem Kontrast zu anderen möglichen, aber nicht gewählten Kategorisierungen und Beschreibungen für das rhetorische Potenzial und die Handlungsrelevanz ihrer Darstellung zukommt (Edwards 1997). Sie trifft sich dabei durchaus mit manchen konversationsanalytischen Untersuchungen, wie auf Orte (Schegloff 1972) und Personen (Enfield \& Stivers 2007; Schegloff 1996) Bezug genommen wird, sowie mit den von Sacks ,neben“ und teilweise auch im Kontext der KA unter dem Titel der membership categorization analysis durchgeführten Analysen (s. Abschnitt 5.1). Die interaktive Konstruktion von Darstellungen wurde in der KA vor allem in Bezug auf die situations-, sequenz- und sprecher/innenrollengebundenen Funktionen von Reformulierungen (formulations, Heritage \& Watson 1979; Drew 2003) untersucht. Dabei geht es darum, wie eine erste Version eines Sachverhalts, die ein vorangehender Sprecher/eine vorangehende Sprecherin formuliert hatte, nachfolgend paraphrasiert, zusammengefasst oder in Bezug auf Schlussfolgerungen ausgedeutet wird.

\section{Beiträge zu klassischen psychologischen Fragestellungen}

\subsection{Identitäten in der Interaktion}

Neben der sequenziellen Organisation von Interaktionen befasste sich Sacks vor allem mit Fragen der membership categorization analysis (MCA, s. Sacks 1992). Die MCA untersucht die Systematik der Verwendung und Interpretation von sozialen Kategorisierungen durch Gesellschaftsmitglieder. Seit Mitte der 1990er Jahre werden derartige Fragen sowohl von Konversationsanalytiker/innen als auch von diskursiven Psycholog/innen verstärkt unter dem Titel identities in talk bearbeitet (Antaki \& Widdicombe 1998; Benwell \& Stokoe 2006, Kap. 2-3). Hier geht es bspw. darum, wer aufgrund welcher Kriterien als Mitglied einer Kategorie behandelt wird, welche Rechte und Pflichten Kategorienmitgliedern zugeschrieben werden, wie Kategorisierungen eingesetzt werden, um Handlungen zu erklären und zu rechtfertigen, wie aus Handlungen Schlussfolgerungen über Kategorienmitgliedschaft gezogen werden usw. In diesen Forschungen wird die Relevanz sozialer Identitäten für die Gesellschaftsmitglieder selbst anhand ihrer Verwendung in der Interaktion erforscht. Im Unterschied zur sozialpsychologischen Theorie der sozialen Identität (Tajfel 1981) werden Gruppenmitgliedschaften nicht als statische, bedingende Faktoren für soziales Handeln verstanden, sondern als symbolische Größen, die Interaktionsteilnehmende selbst in- 
terpretieren und für die Konstitution ihrer Handlungen in Anspruch nehmen. Dabei zeigt sich, dass Identitäten keineswegs Handeln determinieren, sondern dass die Relevanz vs. Irrelevanz einzelner Identitätsaspekte situativ variiert und interaktiv ausgehandelt wird, und dass es hinsichtlich des Rechts zur Zuschreibung und Interpretation bestimmter (z.B. subkultureller) Identitätskategorien unterschiedliche Autorisierungen gibt, je nachdem, zu welcher Kategorie der Sprecher/die Sprecherin selbst gehört (Widdicombe \& Wooffitt 1995), sodass die Frage nach der authentischen Inanspruchnahme von Identitätskategorien ein Feld für Konflikte und symbolische Machtkämpfe werden kann. Darüber hinaus besteht die Inanspruchnahme und Verhandlung von Identität in Interaktionen auch in Handlungen des doing being $X$, d.h., der performativen Darstellung und Zuweisung bestimmter Identitäten durch entsprechende kategoriengebundene Handlungen, ohne dass dies in Form expliziter Selbst- oder Fremdkategorisierung geschehen muss. Als umfassender Begriff für konversationelle Identitätspraktiken erlaubt es der Begriff der Positionierung (Davies \& Harré 1990; Bamberg 1997; Lucius-Hoene \& Deppermann 2004a, 2004b), die Formen zu untersuchen, mit denen Identitäten in der Interaktion kategorial, deskriptiv und performativ relevant gemacht und verhandelt werden. Der Blick auf Interaktionen als Schauplatz der Entfaltung und Verhandlung von Identitäten steht in mehrfacher Weise zu den in psychologischen Ansätzen gängigen Identitätsvorstellungen im Gegensatz:

- Anstelle eines integrativ übersituativen Identitätskonzepts werden die dynamischen, situativ fluktuierenden Prozesse der Identitätszuschreibung untersucht.

- Der Fokus verschiebt sich von entweder sozialstrukturell verbürgten „objektiven“ Identitäten (wie in der Theorie der sozialen Identität) bzw. von reflexiv verfügbaren und explizit selbst zugeschriebenen Identitäten (wie in der Selbstkonzeptforschung) hin zu performativen, im Handeln in Anspruch genommenen und enaktierten Identitäten.

- Es wird nicht (wie in der Selbstkonzeptforschung, aber auch in manchen Konzeptionen des qualitativen Interviews) eine mehr oder weniger (vermeintlich) stabile biografische Selbstsicht abgefragt und Identität in der isolierten Person lokalisiert, sondern Identität wird als in der Interaktion verhandelte Größe und als ihr kollektives, manchmal auch umstrittenes Produkt untersucht.

- Es interessiert nicht wie in der sozialwissenschaftlichen Biografieforschung die Langzeit- und Prozessperspektive des Werdens der Person, sondern die funktionale Konstitution von Identität im Hier und Jetzt in Bezug auf bestimmte Gesprächsaufgaben und Gesprächspartner/innen in flüchtigen Interaktionen.

Diese veränderten Akzentsetzungen in der Sicht von Identität werden maßgeblich im Konzept der small stories (Bamberg \& Georgakopoulou 2008; Georgakopoulou 2007) erfasst, welches konversationsanalytische, diskursiv-psychologische und narratologische Ansätze der Identitätsanalyse zusammenführt. Eine vergleichbare Identitätskonzeption vertreten Lucius-Hoene und Deppermann (2004a) für die Analyse „klassischer“ narrativer Interviews, die nicht mehr primär als Basis der Rekonstruktion biografischer Prozessstrukturen, sondern als besonders reichhaltige Situationen der Identitätskonstitution durch das narrative Management verschiedener zeitlicher und interpersoneller Ebenen durch den Erzähler/die Erzählerin analysiert werden. 


\subsection{Kognition in Interaktion}

Die DP wendet sich gegen die in der Psychologie gängige Auffassung, dass verbale Darstellungen in Form von Kategorisierungen, Berichten oder Erzählungen (z.B. von Handlungen in Interviews, Selbstzuschreibung von Identitätsmerkmalen in Fragebögen) ein mehr oder weniger transparentes Fenster auf dahinterliegende psychologische Sachverhalte (wie Emotionen, Kognitionen, Identitäten, Einstellungen) seien (Potter et al. 1993; Edwards 1997; Edwards \& Potter 2005). Anstatt also aus verbalen Daten auf Psychisches zu schließen und anstatt Annahmen über Psychisches zu benutzen, um Interaktionshandeln zu erklären, untersucht die DP, wie mit mentalen Prädikaten und mit verbalen Darstellungen, in denen Psychisches thematisiert wird (z.B. mentale Termini wie ich denke, ich weiß nicht; Berichte über Absichten oder Erinnerungen), soziale Handlungen vollzogen werden. Die Frage ist für die DP also nicht, wie und welche psychische Realitäten (z.B. autobiografische Erinnerungen) verbal abgebildet werden und ob diese Abbildungen valide sind, sondern welches rhetorisch-diskursfunktionale Potenzial Darstellungen mentaler Phänomene und ganz allgemein die Begrifflichkeiten für Mentales in der Interaktion besitzen. Edwards (1997) argumentiert z.B., dass die Formulierung von scripts und die Unterstellung von common ground nicht einfach kognitive Gegebenheiten widerspiegeln, sondern dass es sich um rhetorische Verfahren handelt, mit denen die Normalität und Gewissheit der eingenommenen Positionen und Bewertungen argumentativ abgesichert und gewissermaßen sozial verpflichtend gemacht wird.

Die DP nimmt in Bezug auf das Verhältnis von Kognition und Interaktion eine strikt konstruktivistische, anti-essentialistische Sicht kognitiver Größen ein. Sie hält es aus methodologischen Erwägungen grundsätzlich für nicht statthaft, ja vielleicht gar für zirkulär und scheinhaft, aus Interaktionshandeln auf kognitive Gegebenheiten zu schließen. Die DP ist also zumindest methodologisch, wenn nicht gar ontologisch, antimentalistisch. Einige KA-Forschende sind dagegen sehr wohl bereit, bestimmte Interaktionsphänomene als konventionellen Ausdruck mentaler Zustände wie Verwirrung (Drew 2005), neu gewonnenen Verständnisses (Heritage 2005b) oder von Planungen und Absichten (Drew 1995) zu analysieren (vgl. auch die Diskussion in te Molder \& Potter 2005). Zunehmend richtet sich das Interesse der KA dabei auf die Verhandlung von Wissensansprüchen und -zuschreibungen in der Interaktion (Heritage \& Raymond 2005).

\subsection{Psychotherapiegespräche}

Ein jüngst erschienener Sammelband dokumentiert den aktuellen Stand der konversationsanalytischen Forschung zur psychotherapeutischen Interaktion (Peräkylä, Antaki, Vehviläinen \& Leudar 2008). Die Methodik der KA ist besonders geeignet, dem oftmals beklagten Defizit an Prozessforschung im Unterschied zur vorherrschenden Outcome-Forschung abzuhelfen und die interaktiven Prozesse in Therapien in einer schulenunabhängigen Weise auf der Grundlage einer allgemeinen Methodologie zur Untersuchung verbaler Interaktionen $\mathrm{zu}$ beschreiben. Die Beschreibungsgenauigkeit der KA ist dabei den traditionellen Formen der Prozessdokumentation durch Notizen, Gedächtnisprotokolle oder die Kodierung von Videoaufnahmen überlegen, da hier der sequenzielle Prozess der Interaktion zwischen Therapeut/in und Patient/in in Bezug auf ihre verbale, aber auch nonverbale Koordi- 
nation Moment für Moment nachgezeichnet werden kann und so die sprachliche und körperliche Enaktierung von Beziehungsmustern zu untersuchen ist (Streeck 2004). Die KA kann aus psychologischer Sicht genutzt werden, um den Gehalt psychotherapietheoretischer Konstrukte wie „Interpretation“, ,,aktives Zuhören“, „Widerstand“ in Bezug auf die faktischen Abläufe von Therapiegesprächen auf den Prüfstand zu stellen, da zumeist unklar ist, wie ihre sprachlich-kommunikative Realisierung in konkreten Therapiegesprächskontexten aussieht, wann sie produziert werden und welche Reaktionen sie hervorrufen. So wurde z.B. untersucht, wie Therapeut/innen gezielt Wortersetzungen benutzen, um den Patient/innen auf den latenten emotionalen Gehalt ihrer Erzählungen zu fokussieren, oder wie sie durch Reformulierungen der Patient/innenäußerungen deren psychologisch bzw. therapeutisch relevanten Aspekte fokussieren, den Patient/innen als das von ihnen Gemeinte widerspiegeln und damit die Grundlage für die weitere Interaktion strategisch im Sinne der Katalysierung therapeutisch intendierter Effekte modifizieren.

\section{Stärken, Schwächen und Desiderata}

Soziale Interaktionen sind der Stoff, aus dem ein Großteil unseres Alltagslebens gestrickt ist und der unser Schicksal maßgeblich bestimmt, aber sie werden in der Psychologie kaum in ihrer natürlichen, alltagsweltlichen Phänomenologie untersucht. Dies verwundert nicht, kommt es doch bei der Analyse von Interaktion als Interaktion auf genau das an, was die experimental-psychologische Methodenlehre gerade zu eliminieren sucht: Alltagsnähe (vs. Bedingungskontrolle), Kontextabhängigkeit (vs. Standardisierung), prinzipielle Unabschließbarkeit der Handlungsoptionen (vs. geschlossene Variablensets) und prozessrelative Interpretation (vs. kontextfreie Codierung). KA und DP sind Ansätze, deren Methodologie auf genau diese Eigenschaften des Untersuchungsgegenstands zugeschnitten ist. Ihr Datenverständnis fordert und kultiviert eine methodologische Disziplin des genauen Hinhörens und -sehens und damit eine strikt phänomenologische Orientierung auf den Fall, die gerade auch klinisch relevant ist. Ihr Ansatz, sich theoretisch unvoreingenommen der rigorosen Prüfung aller Hypothesen an den Details des Interaktionsprozesses zu stellen und die wissenschaftlichen Kategorien im Handeln und damit im Selbstverständnis der Interaktionteilnehmenden selbst zu verankern, beinhaltet ein großes Potenzial für eine breite Palette von Fragestellungen aus Sozial-, Kognitions- und Sprachpsychologie und für die Therapieforschung.

Viele Psycholog/innen dürften es als einen Mangel empfinden, dass KA und DP groBen Aufwand in die detailgenaue Beschreibung von Interaktionspraktiken und in die Analyse ihrer Funktionen investieren, Fragen der Erklärung und Vorhersage - warum benutzt wer wann welche Praktiken? - dagegen weitgehend ausklammern. Dies ist eine Folge des ethnomethodologisch inspirierten Erkenntnisinteresses, das seine Grenze dort findet, wo für das Interaktionshandeln andere Größen als die an ihm selbst ausweisbaren Orientierungen der Teilnehmenden mit maßgeblich sind. Erste Ansätze zur Integration von KA und quantitativen Untersuchungen, in denen Eigenschaften der Interaktion mit Kontextvariablen korreliert werden, liegen vor. Die Verknüpfung gestaltet sich aber schwierig, will man damit nicht zugleich die methodologischen Vorteile von KA und DP durch Desequenzialisierung und deduktive Kodierung wieder zunichte machen. Dennoch bestehen hier Perspektiven für 
die Entwicklung von Mixed-Method-Ansätzen, ein Vorhaben, das allerdings unter Vertreter/innen der KA und DP sehr umstritten ist.

Auch Forschende anderer qualitativer Couleur, insbesondere aus der kritischen Diskursanalyse, der objektiven Hermeneutik, der Ethnografie und den Gender Studies haben Vorbehalte gegen KA und DP, da sie zu wenig Kontext (nämlich nur den gesprächsinternen) einbezögen, die Einbettung in größere sozialstrukturelle oder auch psychobiografische Zusammenhänge vernachlässigten und deshalb tendenziell naiv und positivistisch an der Oberfläche der Daten klebten. In vielen Fällen ist zu zeigen, dass diese Kritik gegenstandslos ist, da im Gegenteil die Berufung auf gesprächsexterne Kontexte leicht vorschnell geschieht, zu voreingenommenen Scheinerklärungen führt und stattdessen versäumen lässt, die interaktionseigenen Motivationen kommunikativer Phänomene $\mathrm{zu}$ erkennen. Es lässt sich aber nicht leugnen, dass für gewisse Phänomene und Fragestellungen der strikt interaktionsanalytische Forschungsrahmen der KA und DP bspw. um interaktionstranszendente ethnografische Daten und Wissensbestände erweitert werden muss (s. Deppermann 2000), und dass trotz aller Meriten der rhetorisch-funktionalen Sicht auf Kognitionen die Position der DP, auf Kognitionszuschreibungen in der Analyse verzichten zu können, letzten Endes aporetisch ist (Deppermann 2010).

In der Verknüpfung mit quantitativen Studien, der Ethnografisierung und dem Einbezug und der Relationierung interaktiver zu kognitiven Größen liegen also Potenziale für die künftige Weiterentwicklung von KA und DP.

\section{Weiterführende Literatur}

Deppermann, Arnulf (1999). Gespräche analysieren. Opladen: Leske + Budrich. Hutchby, Ian \& Wooffitt, Robin (2008). Conversation analysis (2. Aufl.). Cambridge: Polity Press. Levinson, Stephen C. (1990). Pragmatik. Kap.6. Tübingen: Niemeyer.

\section{Literatur}

Antaki, Charles \& Widdicombe, Sue (Hrsg.) (1998). Identities in talk. London: Sage.

Atkinson, John Maxwell \& Drew, Paul (1979). Order in court. The organisation of verbal interaction in judicial settings. London: Macmillan.

Atkinson, John Maxwell \& Heritage, John (Hrsg.) (1984). Structures of social action. Studies in conversation analysis. Cambridge, MA: Cambridge University Press.

Auer, Peter (1999). Sprachliche Interaktion. Tübingen: Niemeyer.

Auer, Peter (2005). Projection in interaction and projection in grammar. Text 25(1), 7-36.

Bamberg, Michael (1997). Positioning between structure and performance. Journal of Narrative and Life History, 7, 335-342.

Bamberg, Michael \& Georgakopoulou, Alexandra (2008). Small stories as a new perspective in narrative and identity analysis. Text \& Talk, 28(3), 377-396.

Benwell, Bethan \& Stokoe, Elisabeth H. (2006). Discourse and identity. Edinburgh: University Press.

Bergmann, Jörg R. (1981). Ethnomethodologische Konversationsanalyse. In Peter Schröder \& Hugo Steger (Hrsg.), Dialogforschung. Jahrbuch des Instituts für deutsche Sprache 1980 (S.9-51). Düsseldorf: Pädagogischer Verlag Schwann. 
Bergmann, Jörg R. (1982). Schweigephasen im Gespräch - Aspekte ihrer interaktiven Organisation. In Hans-Georg Soeffner (Hrsg.), Beiträge zu einer empirischen Sprachsoziologie (S.143-183). Tübingen: Gunter Narr.

Bergmann, Jörg R. (1985). Flüchtigkeit und methodische Fixierung sozialer Wirklichkeit: Aufzeichnungen als Daten interpretativen Soziologie. In Wolfgang Bonß \& Heinz Hartmann (Hrsg.), Entzauberte Wissenschaft. Zur Relativität und Geltung soziologischer Forschung (S.299-320). Göttingen: Schwartz.

Bergmann, Jörg R. (1987). Klatsch. Zur Sozialform der diskreten Indiskretion. Berlin: de Gruyter. Birkner, Karin (2001). Bewerbungsgespräche mit West- und Ostdeutschen. Tübingen: Niemeyer.

Clayman, Steven E. \& Heritage, John (2002). The news interview. Cambridge, MA: Cambridge University Press.

Couper-Kuhlen, Elizabeth \& Ford, Cecilia E. (Hrsg.) (2004). Sound patterns in interaction. Crosslinguistic studies from conversation. Amsterdam: John Benjamins.

Davies, Bronwyn \& Harré, Rom (1990). Positioning. The discursive production of selves. Journal for the Theory of Social Behaviour, 20, 43-63.

Deppermann, Arnulf (1997). Glaubwürdigkeit im Konflikt. Frankfurt/M.: Peter Lang.

Deppermann, Arnulf (1999). Gespräche analysieren. Opladen: Leske + Budrich.

Deppermann, Arnulf (2000). Ethnographische Gesprächsanalyse: Zu Nutzen und Notwendigkeit von Ethnographie für die Konversationsanalyse. Gesprächsforschung, 1, 96-124, http://www. gespraechsforschung-ozs.de/heft2000/heft2000.htm.

Deppermann, Arnulf (2007). Grammatik und Semantik aus gesprächsanalytischer Sicht. Berlin: de Gruyter.

Deppermann, Arnulf (2008). Verstehen im Gespräch. In Heidrun Kämper \& Ludwig M. Eichinger (Hrsg.), Sprache - Kognition - Kultur. Jahrbuch des Instituts für Deutsche Sprache 2007 (S.225-261). Berlin: de Gruyter.

Deppermann, Arnulf (2010). How does „cognition“ matter to the analysis of talk-in-interaction? In Language Sciences.

Deppermann, Arnulf \& Schmitt, Reinhold (2007). Koordination. Zur Begründung eines neuen Forschungsgegenstandes. In Reinhold Schmitt (Hrsg.), Koordination. Analysen zur multimodalen Interaktion (S. 15-54). Tübingen: Narr.

Deppermann, Arnulf \& Schütte, Wilfried (2008). Data and transcription. In Gerd Antos, Eija Ventola \& Tilo Weber (Hrsg.), Handbook of interpersonal communication (S.179-213). Berlin: Mouton de Gruyter.

Drew, Paul (1995). Interaction sequences and anticipatory interactional planning. In Esther Goody (Hrsg.), Social intelligence and interaction. Expressions and implications of the social bias in human intelligence (S.111-138). Cambridge: Cambridge University Press.

Drew, Paul (2003). Comparative analysis of talk-in-interaction in different institutional settings. In Philipp J. Glenn, Curtis D. LeBaron, \& Jenny Mandelbaum (Hrsg.), Studies in language and social interaction (S.293-308). Mahwah, NJ: Lawrence Erlbaum,.

Drew, Paul (2005). Is confusion a state of mind? In Hedwig te Molder \& Jonathan Potter (Hrsg.), Conversation and cognition (S.161-183). Cambridge: Cambridge University Press.

Drew, Paul \& Heritage, John (Hrsg.) (1992). Talk at work. Cambridge: Cambridge University Press.

Edwards, Derek (1997). Discourse and cognition. London: Sage.

Edwards, Derek \& Potter, Jonathan (2005). Discursive psychology, mental states and descriptions. In Hedwig te Molder \& Jonathan Potter (Hrsg.), Conversation and cognition (S.241-259). Cambridge: Cambridge University Press.

Edwards, Derek \& Potter, Jonathan (1992). Discursive psychology. London: Sage.

Egbert, Maria (2009). Der Reparatur-Mechanismus in deutschen Gesprächen. Mannheim: Verlag für Gesprächsforschung, http://www.verlag-gespraechsforschung.de/2009/egbert.htm.

Enfield, Nick \& Stivers, Tanya (Hrsg.) (2007). Person reference in interaction. Cambridge: Cambridge University Press.

Garfinkel, Harold (1967). Studies in ethnomethodology. Englewood Cliffs NJ: Prentice-Hall. 
Garfinkel, Harold \& Sacks, Harvey (1976). Über formale Strukturen praktischer Handlungen. In Elmar Weingarten, Fritz Sack \& Jim Schenkein (Hrsg.), Ethnomethodologie. Beiträge zu einer Soziologie des Alltagshandelns (S.130-176). Frankfurt/M.: Suhrkamp.

Georgakopoulou, Alexandra (2007). Small stories, Interaction and identities. Amsterdam: John Benjamins.

Glaser, Barney G. \& Strauss, Anselm L. (1967). The discovery of grounded theory. Strategies for qualitative research. New York, NY: Aldine.

Goffman, Erving (1971). Interaktionsrituale. Über Verhalten in direkter Kommunikation. Frankfurt/M.: Suhrkamp.

Goffman, Erving (1974). Das Individuum im öffentlichen Austausch. Frankfurt/M.: Suhrkamp.

Goffman, Erving (1981). Footing. In Erving Goffman (Hrsg.), Forms of talk (S.124-159). Philadelphia: University of Pennsylvania Press.

Günthner, Susanne \& Knoblauch, Hubert (1994). „Forms are the food of faith“. Gattungen als Muster kommunikativen Handelns. Kölner Zeitschrift für Soziologie und Sozialpsychologie, 4, 693-723.

Gumperz, John J. (1982). Discourse strategies. Cambridge: Cambridge University Press.

Heath, Christian, Hindmarsh, Jon \& Luff, Paul (2010). Video in qualitative research. London:Sage.

Heritage, John (1984). Garfinkel and ethnomethodology. Oxford: Polity.

Heritage, John (1995). Conversation analysis. Methodological aspects. In Uta M. Quasthoff (Hrsg.), Aspects of oral communication (S.391-418). Berlin: de Gruyter.

Heritage, John (2005a). Conversation analysis and institutional talk. In Kristine L. Fitch \& Robert E. Sanders (Hrsg.), Handbook of language and social interaction (S.103-147). Mahwah NJ: Erlbaum.

Heritage, John (2005b). Cognition in discourse. In Hedwig te Molder \& Jonathan Potter (Hrsg.), Conversation and cognition (S.184-202). Cambridge: Cambridge University Press.

Heritage, John \& Clayman, Steven (2002). The new interview. Cambridge: Cambridge University Press.

Heritage, John \& Maynard, Douglas W. (Hrsg.) (2006). Communication in medical care: Interaction between primary care physicians and patients. Cambridge: Cambridge University Press.

Heritage, John \& Raymond, Geoffrey (2005). The terms of agreement. Indexing epistemic authority and subordination in talk-in-interaction. Social Psychology Quarterly, 68, 15-38.

Heritage, John \& Watson, Rod (1979). Formulations as conversational objects. In George Psathas (Hrsg.), Everyday language (S.123-162). New York: Irvington.

Hutchby, Ian \& Wooffitt, Robin (2008). Conversation analysis (2. Aufl.). Cambridge: Polity Press.

Kallmeyer, Werner (1985). Handlungskonstitution im Gespräch: Dupont und sein Experte führen ein Beratungsgespräch durch. In Elisabeth Gülich \& Thomas Kotschi (Hrsg.), Grammatik, Konversation, Interaktion (S.81-123). Tübingen: Niemeyer.

Kallmeyer, Werner (2005). Konversationsanalytische Beschreibung. In Ulrich Ammon, Norbert Dittmar \& Klaus J. Mattheier (Hrsg.), Soziolinguistik / Sociolinguistics (Bd. 2, S.1212-1225). Berlin: de Gruyter.

Kallmeyer, Werner \& Schütze, Fritz (1976). Konversationsanalyse. Studium Linguistik 1, 1-28.

Kallmeyer, Werner \& Schütze, Fritz (1977). Zur Konstitution von Kommunikationsschemata der Sachverhaltsdarstellung - Exemplifiziert am Beispiel von Erzählungen und Beschreibungen. In Dirk Wegner (Hrsg.), Gesprächsanalysen (S.159-274). Hamburg: Buske.

Knoblauch, Hubert \& Heath, Christian (1999). Technologie, Interaktion und Organisation: die Workplace Studies. Schweizerische Zeitschrift für Soziologie, 25(2), 163-181.

Levinson, Stephen C. (1990). Pragmatik. Tübingen: Niemeyer.

Levinson, Stephen C. (2005). Living with Manny's dangerous idea. Discourse Studies, 7(4-5), 431453.

Lucius-Hoene, Gabriele \& Deppermann, Arnulf (2004a). Rekonstruktion narrativer Identität. Wiesbaden: VS Verlag für Sozialwissenschaften. 
Lucius-Hoene, Gabriele \& Deppermann, Arnulf (2004b). Narrative Identität und Positionierung. Gesprächsforschung, 5, 166-183, http://ww.gespraechsforschung-ozs.de/heft2004/heft2004.htm.

Martini, Mareike (2008). Deutsch-kubanische Arbeitsbesprechungen. Tübingen: Stauffenburg.

Mondada, Lorenza \& Schmitt, Reinhold (Hrsg.) (2010). Situationseröffnungen. Tübingen: Narr.

Neises, Mechthild; Ditz, Susanne \& Spranz-Fogasy, Thomas (Hrsg.) (2005). Psychosomatische Gesprächsführung in der Frauenheilkunde. Stuttgart: Wissenschaftliche Verlagsgesellschaft.

Nothdurft, Werner; Reitemeier, Ulrich \& Schröder, Peter (1994). Beratungsgespräche. Tübingen: Narr.

Peräkylä, Anssi; Antaki, Charles; Vehviläinen, Sanna \& Leudar, Ivan (Hrsg.) (2008). Conversation analysis and psychotherapy. Cambridge: Cambridge University Press.

Pomerantz, Anita (1984). Agreeing and disagreeing with assessments. Some features of preferred / dispreferred turn shapes. In John Maxwell Atkinson \& John Heritage (Hrsg.), Structures of social action (S.57-101). Cambridge: Cambridge University Press.

Potter, Jonathan (1996). Representing reality. London: Sage.

Potter, Jonathan \& Wetherell, Margaret (1987). Discourse and social psychology: Beyond attitudes and behaviour. London: Sage.

Potter, Jonathan; Edwards, Derek \& Wetherell, Margaret (1993). A model of discourse in action. American Behavioral Scientist, 36(3), 83-401,

Psathas, George (1995). Conversation analysis. London: Sage.

Sacks, Harvey (1984). Notes on methodology. In John Maxwell Atkinson \& Heritage, John (Hrsg.), Structures of social action (S.21-27). Cambridge: Cambridge University Press.

Sacks, Harvey \& Jefferson, Gail (Hrsg.) (1992). Lectures on conversation. Vol. 2. Oxford: Blackwell.

Sacks, Harvey; Schegloff, Emanuel A. \& Jefferson, Gail (1974). A simplest systematics for the organization of turn-taking in conversation. Language, 50(4), 696-735.

Saville-Troike, Muriel (1989). The ethnography of communication (2. Aufl.). Oxford: Blackwell.

Schegloff, Emanuel A. (1968). Sequencing in conversational openings. American Anthropologist, 70, 1075-1095.

Schegloff, Emanuel A. (1972). Notes on a conversational practice: Formulating place. In David Sudnow (Hrsg.), Studies in social interaction (S.75-119). New York: Free Press.

Schegloff, Emanuel A. (1991). Reflections on talk and social structure. In Deirdre Boden \& Don H. Zimmerman (Hrsg.), Talk and social structure (S.44-71). Cambridge, England: Polity.

Schegloff, Emanuel A. (1992). Repair after next turn. The last structurally provided defence of intersubjectivity in conversation. American Journal of Sociology, 97(5), 1295-1345.

Schegloff, Emanuel A. (1996). Some practices of referring to persons in talk-in-interaction. A partial sketch of a systematics. In Barbara A. Fox (Hrsg.), Studies in anaphora (S.437-485). Amsterdam: John Benjamins.

Schegloff, Emanuel A. (1997). Practices and actions. Boundary cases of other-initiated repair. Discourse Processes, 23(3), 499-545.

Schegloff, Emanuel A. (1997). Whose text? Whose context? Discourse and Society, 8, 165-187.

Schegloff, Emmanuel A. (2005). On integrity in inquiry ... of the investigated, not the investigator. Discourse Studies, 7(4-5), 455-80.

Schegloff, Emanuel A. (2007). Sequence organization in interaction. Cambridge: Cambridge University Press.

Schegloff, Emanuel A. \& Sacks, Harvey (1973). Opening up closings. Semiotica, 8(4), 289-327.

Schegloff, Emanuel A., Jefferson, Gail \& Sacks, Harvey (1977). The preference for self-correction in the organization of repair in conversation. Language, 53(2), 361-382.

Schmitt, Reinhold (Hrsg.) (2007). Koordination. Analysen zur multimodalen Interaktion. Tübingen: Narr.

Schütz, Alfred (1981[1932]). Der sinnhafte Aufbau der sozialen Welt. Frankfurt/M.: Suhrkamp.

Searle, John R. (1971). Sprechakte. Frankfurt/M.: Suhrkamp. 
Selting, Margret \& Couper-Kuhlen, Elizabeth (2000). Argumente für die Entwicklung einer ,interaktionalen Linguistik". Gesprächsforschung. Online-Zeitschrift zur verbalen Interaktion, 1, 76-95, http://www.gespraechsforschung-ozs.de/heft2000/heft2000.htm.

Selting, Margret; Auer, Peter; Barth-Weingarten, Dagmar; Bergmann, Jörg \& Bergmann, Pia et al. (2009). Gesprächsanalytisches Transkriptionssystem 2 (GAT 2). Gesprächsforschung, 10, http://www.gespraechsforschung-ozs.de/heft2009/heft2009.htm.

Streeck, Ulrich (2004). Auf den ersten Blick. Psychotherapeutische Beziehungen unter dem Mikroskop. Stuttgart: Klett-Cotta.

Tajfel, Henri (1981). Human groups and social categories. Cambridge: Cambridge University Press.

te Molder, Hedwig \& Potter, Jonathan (Hrsg.) (2005). Conversation and cognition. Cambridge: Cambridge University Press.

Wadensjö, Cecilia (1992). Interpreting as interaction. Linköping: University of Linköping.

Wetherell, Margaret (1998). Positioning and interpretive repertoires. Conversation analysis and poststructuralism in dialogue. Discourse and Society, 9, 387-412.

Widdicombe, Sue \& Wooffitt, Robin (1995). The language of youth subcultures: Social identity in action. Hempel Hempstead: Harvester Wheatsheaf.

Wooffitt, Robin (1992). Telling tales of the unexpected. The organization of factual discourse. Hempel Hempstead: Harvester Wheatsheaf.

Wooffitt, Robin (2005). Conversation analysis and discourse analysis: A comparative and critical introduction. London: Sage. 